\title{
A closed model for the respiratory system in mammals
}

\author{
Choongseok Park ${ }^{1 *}$, Yaroslav Molkov², Alona Ben-Tal ${ }^{3}$, Natalia Shevtsova ${ }^{4}$, Jeffrey Smith ${ }^{5}$, llya Rybak ${ }^{4}$, \\ Jonathan Rubin ${ }^{1}$ \\ From Twenty First Annual Computational Neuroscience Meeting: CNS*2012 \\ Decatur, GA, USA. 21-26 July 2012
}

The interactions between rhythmogenic neuronal networks and physiological systems are crucial components in understanding how a system maintains homeostasis under diverse circumstances. Various aspects of such interactions should be addressed including how rhythms are generated in neuronal networks, how feedback signals interact with neuronal networks to regulate levels of important physiological quantities, and how changes in demands on the system can be handled in a way that restores homeostasis.

In this work, we explore these issues in the context of neuronal networks in the mammalian brainstem that generate the respiratory rhythm when isolated from the physiological systems that drive actual breathing [1]. These networks include neuronal components within the Bötzinger (BötC) and pre-Bötzinger (pre-BötC) complexes and the retrotrapezoid nucleus/parafacial respiratory group (RTN/pFRG). Experimental results on respiratory rhythm generation have suggested that neurons within the BötC and pre-BötC are responsible for the generation of primary respiratory rhythms, which are modulated by oscillations in the RTN/pFRG. Previous computational work has elucidated a possible set of mechanisms through which these brain stem neurons interact and produce a variety of experimental results [2].

Separate past work has developed a physiologically detailed yet tractable model of respiratory physiology [3]. This model is comprised of a very simplified version of a neuronal network oscillator that generates respiratory rhythms, an integrator that delivers respiratory output , and lung components responsible for air exchange. This earlier study considered reasonable ways to link the

\footnotetext{
*Correspondence: cspark@pitt.edu

'Department of Mathematics, University of Pittsburgh, Pittsburgh, PA15260, USA

Full list of author information is available at the end of the article
}

neuronal oscillator/integrator with these physiological components in a closed loop feedback system to achieve respiratory frequency and amplitude control.

In our work, we simulate and analyze a model that links the detailed neuronal rhythm generator with the physiological lung components. We consider the roles of mechanical and chemoreceptive feedback signals in maintaining stable respiratory rhythms and in responding to metabolic or environmental perturbations. In particular, we study the response to hypercapnia, or excessive carbon dioxide [4]. The idea is that excessive exposure to $\mathrm{CO} 2$ may lead to $\mathrm{CO} 2$ accumulation in the blood, which will recruit certain neuronal elements in the RTN/pFRG and alter the drive to core rhythmogenic elements. Changes in rhythmic output patterns activate certain muscles that alter breathing in a way that restores $\mathrm{CO} 2$ homeostasis. We analyze the roles of neurons' intrinsic dynamic features, the architecture and strengths of synaptic connections among neurons, and the roles of particular feedback systems in achieving baseline rhythms and appropriate responses to perturbations.

\section{Acknowledgements \\ This work is done in collaboration with Alona Ben-Tal, llya Rybak, Natalia Shevtsova, and Jeffrey Smith.}

\section{Author details}

'Department of Mathematics, University of Pittsburgh, Pittsburgh, PA15260, USA. ${ }^{2}$ Indiana University - Purdue University Indianapolis, Department of Mathematical Sciences, Indianapolis, IN 46202, USA. ${ }^{3}$ Institute of Information and Mathematical Sciences, Massey University, Auckland, New Zealand. ${ }^{4}$ Drexel University College of Medicine, Philadelphia, PA 19129, USA. ${ }^{5}$ Cellular and Systems Neurobiology Section, Laboratory of Neural Control, NINDS, Bethesda, MD 20892-3700, USA.

Published: 16 July 2012 


\section{References}

1. Feldman $J L$, Del Negro CA: Looking for inspiration: new perspectives on respiratory rhythm. Nat Rev Neurosci 2006, 7:232-42.

2. Rubin JE, Bacak BJ, Molkov YI, Shevtsova NA, Smith JC: Interacting oscillations in neural control of breathing: modeling and qualitative analysis. J Comput Neurosci 2011, 30:607-632.

3. Ben-Tal A, Smith JC: A model for control of breathing in mammals: coupling neural dynamics to peripheral gas exchange and transport. J Theor Biol 2008, 251:480-97.

4. Abdala AP, Rybak IA, Smith JC, Paton JF: Abdominal expiratory activity in the rat brainstem-spinal cord in situ: patterns, origins and implications for respiratory rhythm generation. J Physiol 2009, 587:3539-59.

doi:10.1186/1471-2202-13-S1-P51

Cite this article as: Park et al:: A closed model for the respiratory system in mammals. BMC Neuroscience 2012 13(Suppl 1):P51.

\section{Submit your next manuscript to BioMed Central} and take full advantage of:

- Convenient online submission

- Thorough peer review

- No space constraints or color figure charges

- Immediate publication on acceptance

- Inclusion in PubMed, CAS, Scopus and Google Scholar

- Research which is freely available for redistribution

Submit your manuscript at www.biomedcentral.com/submit 